\title{
SIMULATION OF COPLANAR PROXIMITY CHARGE SENSING ELECTRODES IN CZT DETECTORS
}

\author{
Fouad A. Abolaban ${ }^{1}$, Abdulsalam M. Alhawsawi ${ }^{1}$ and Andrew Nisbet ${ }^{2}$ \\ 1 King Abdulaziz University, College of Engineering, Nuclear Engineering Department, Jeddah, \\ Kingdom of Saudi Arabia, Jeddah 21589, Saudi Arabia \\ 2 Department of Medical Physics \& Biomedical Engineering, University College London, Malet \\ Place Engineering Building, London WC1E 6BT, UK \\ (e-mail: fabolaban@kau.edu.sa).
}

\begin{abstract}
The concept of applying proximity charge sensing electrodes to semiconductor radiation detectors is a novel technique that has distinct advantages over directly deposited electrodes. This paper evaluates the application of proximity charge sensing onto a CdZnTe (CZT) semiconductor crystal using ANSYS Maxwell simulation software to calculate the weighting potential across the detector depth, the weighting potential across the detector width, and the electric field (E) generated inside the detector volume for multiple designs. To accomplish this goal several variables are studied in the simulated designs including: (1) a high resistivity thin-film material that is applied to the detector proximity surface from the anode side, (2) an appropriate metal that acts as an Ohmic contact to dissipate generated charges on the CZT anode side, and finally (3) an insulating layer (dielectric) to isolate the CZT detector body from the proximity electrodes. The results of the generated weighting potentials for both directly deposited electrodes and proximity-sensing electrodes, having the same electrode width and pitch, have been quantitatively compared using a Figure of Merit (FOM). The FOM compares the weighting potential created by each simulated design for weighting potential uniformity and weighting potential similarity.
\end{abstract}

Keywords: Proximity Charge Sensing, Semiconductor Detectors, CZT crystals, ANSYS Maxell, Weighting Potential, Figure of Merit 


\section{Introduction}

Semiconductor materials have been used in radiation detectors for several decades and provide significantly improved resolution compared to scintillator and gas-based detectors. Broadly speaking, materials used in semiconductor detectors are divided into two main groups: single-element semiconductors, e.g. Si and HPGe, and compound semiconductors, e.g. CdZnTe (CZT) and $\mathrm{HgI}_{2}$. Both types of materials require at least one anode and one cathode electrode, and for an electric field to be applied between these electrodes. Signals are generated when radiation interactions in the crystal generate electron-hole pairs. The electric field then causes the electrons to move towards the positively biased anode and holes to move towards the negatively biased cathode. The movement of these charge carriers induces a current on the external circuit that is integrated to determine the energy deposited by the incident radiation ${ }^{[1,2]}$. These concepts are described in the Shockly-Ramo Theorem, which will be discussed in the next section.

\section{Electrode Types on Semiconductors}

A basic semiconductor detector is made of two simple planar electrodes (low resistivity layers) on either side of a semiconductor crystal. Figure 1 shows radiation interacting with a semiconductor detector that has planar electrodes. The sides without electrodes are covered with a passivation layer to prevent the detector from interaction with the outside environment.

In compound semiconductors such as CZT, holes move 1-2 orders of magnitude more slowly than electrons and are more easily trapped when compared to electrons. This means that the number of holes collected is strongly dependent on the location of the radiation interaction inside the crystal, leading to poor resolution for the basic planar electrode geometry. To overcome this problem, room temperature semiconductors usually employ non-planar electrode patterns that make them almost entirely insensitive to holes ${ }^{[1,2]}$. Semiconductor detectors that use this readout method are referred to as "single-carrier" detectors, since they rely on only one charge carrier (electrons). Examples of common single-carrier detector electrode configurations are the Frisch-Grid, Coplanar-Grid, and Pixelation of the anode electrode. Figure 2 shows coplanar grids. In the figure, electrode 1 is called the cathode. Electrode 2 and electrode 3 are called collecting grid (CG) and non-collecting grid (NCG), respectively. The signal generated on these electrodes is a function of weighting potential $(\varphi)$ for each electrode. The weighting potential will be discussed in the next section.

\section{The Weighting Potential of Coplanar Electrodes}

The total weighting potential generated on the anode of coplanar electrodes is a function of both collecting and non-collecting weighting potentials and is calculated by:

$$
\varphi_{\text {total }}=\varphi_{C G}-\varphi_{N C G}
$$


As stated before, the weighting potential for an electrode is defined as the potential that would exist inside the detector volume when the electrode of interest is set at unit potential (i.e. $V_{\mathrm{CG}}=1$ ) and all other existing grids are set to zero potential (i.e. $\mathrm{V}_{\mathrm{NCG}}=0 \mathrm{~V}$ ) and no space charge exists $[1,2]$.

Traditionally, electrode configurations, whether anodes or cathodes, planar or non-planar, are directly deposited on the crystal surface using metals. Non-planar designs are typically fabricated using photolithography techniques or shadow masks in order to achieve the level of precision required. Thin, delicate wires are then connected to the electrodes and the readout electronics. Direct electrical connection between wires and grids is accomplished using wire bonding, or bump bonding techniques ${ }^{[3,4]}$, which are difficult to fabricate onto fine-pitch anode patterns. Proximity charge sensing can be used to overcome this problem.

Here we use proximity charge sensing, which is a relatively new technique for semiconductor radiation detectors, where the grids or electrodes and the semiconductor crystal are not in direct contact but instead isolated by a dielectric material, usually in thin-film arrangements that are deposited using various methods such as chemical bath deposition ${ }^{[5,6]}$, aerosol-assisted chemical vapor deposition ${ }^{[7]}$, plasma enhanced chemical vapor deposition ${ }^{[8]}$, or Radio Frequency (RF) sputtering ${ }^{[9]}$. Radio Frequency (RF) sputtering will be used to deposit the dielectric material for this work at the implementation stage. Proximity charge sensing has several main advantages:

1. It eliminates the need to directly deposit electrodes on the semiconductor crystal which simplifies the fabrication process and reduces the cost of the whole system,

2. It reduces the leakage current associated with directly depositing the electrodes on the crystal surface,

3. It can be used to improve the position sensitivity of the device via signal interpolation ${ }^{[3,4]}$.

The goal of this work is to simulate multiple designs of coplanar proximity electrodes on a 19.4 mm x $19.4 \mathrm{~mm}$ x $5 \mathrm{~mm}$ CZT crystal. The most promising proximity-sensing electrode design will be recommended to be fabricated, characterized, and its performance compared to directlydeposited electrodes on a similar size crystal.

In 1977, Kurz et al. used proximity electrodes on a position-sensitive HPGe detector ${ }^{[10]}$. The detector used in the experiments was $22 \mathrm{~mm}$ x $13 \mathrm{~mm}$ x $4.4 \mathrm{~mm}$. Figure 3 shows a two-dimensional view of the detector.

In another study, Luke et al. applied the concept of proximity charge sensing in room temperature semiconductor detectors as a means of guiding electrons toward the directly-deposited anode electrodes ${ }^{[11]}$. Figures 4 (a) and 4 (b), show the schematic and weighting potential of the detector respectively. 
Luke et al. modeled a $20 \mathrm{~mm}$ x $20 \mathrm{~mm}$ x $10 \mathrm{~mm}$ detector to calculate the expected weighting potential and the induced charge on a proximity-sensing electrode. Figure 5 (a) and 5 (b) show the geometry and calculated weighting potential of the detector.

A $20 \mathrm{~mm}$ x $20 \mathrm{~mm}$ x $5 \mathrm{~mm} \mathrm{Si}(\mathrm{Li})$ detector was used to test the performance of proximity electrodes experimentally. The $\mathrm{Si}(\mathrm{Li})$ detector had a Li-diffused layer that was washed off and replaced with an amorphous silicon (a-Si) contact similar to that in Luke et als' previous work. The sheet resistance of the a-Si was $\sim 10^{7} \Omega / \square$, which is appropriate for silicon detectors that have a leakage current of $\sim 10 \mathrm{nA}$. An aluminum ring was deposited on the detector surface except for a $10 \mathrm{~mm} \mathrm{x}$ $10 \mathrm{~mm}$ area in the middle left for the proximity surface. The proximity electrode was printed on a PCB and was held at approximately $60 \mu \mathrm{m}$ from the detector surface. Figure 6 shows a schematic of the silicon detector with proximity electrodes.

Figure 7 shows the actual silicon detector with proximity electrodes suspended on top of the detector surface.

The concept of incomplete charge collection-rejection using proximity electrodes was tested using a Germanium $(\mathrm{Ge})$ detector in this work. An induced charge on proximity electrodes (Figure 8) that are placed around the detector side surfaces indicate incomplete charge collection and can be used in anticoincidence mode to reject these signals.

Figure 9 shows the actual Ge detector side surfaces surrounded by proximity electrodes, where two measurements were taken at the same time to generate two spectra while changing the source location.

Figure 10 shows the difference in Compton counts when proximity electrode signals are used in anticoincidence mode to reject incomplete charge collection.

Finally, Proximity-sensing electrodes at the cathode side were implemented by Luke et al. in an HPGe detector ${ }^{[4]}$. The detector used in this study was an $18 \times 18 \times 10 \mathrm{~mm}$ HPGe crystal, coated with amorphous Ge (a-Ge). The a-Ge layer should have a sheet resistivity of at least $10^{9} \Omega / \square$ since Ge detectors have low leakage current. Figure 11 shows the detector schematic. 


\section{Materials and Methods}

The model used to simulate proximity electrodes included all the layers and materials that will be used in the actual system. The coplanar electrode design shown in Figure 12 was used in all ANSYS Maxwell simulations. The pattern was designed to be directly deposited on a CZT crystal for a project by Ranjbar et. al. ${ }^{[12]}$. The reason for using the same pattern is to compare the performance of directly deposited electrodes against proximity electrodes once the proximity design is implemented. Table 1 shows the layers used in the simulation model from the bottom up:

Figure 13 shows a schematic of the layers used in the detector model.

The weighting potentials generated for coplanar proximity electrodes are quantitatively evaluated based on a Figure of Merit (FOM) using two features of the weighting potential across the detector width: uniformity and similarity. Uniformity is a measure of the degree to which the weighting potential for each grid maintains a constant value across the detector width ${ }^{[11]}$.

Uniformity value, $\mathrm{U}$, is calculated individually for both collecting and non-collecting grids using:

$$
U=\sum_{i}\left|\varphi_{i}-\varphi_{m}\right|
$$

where $\varphi_{\mathrm{i}}$ is the weighting potential at location $\mathrm{i}$ across the width of the detector and $\varphi_{\mathrm{m}}$ is the weighting potential in the middle of the detector.

Similarity is a measure of the degree to which the weighting potentials of the collecting and noncollecting grids are equal across the width of the detector $1 \mathrm{~mm}$ below the anode surface ${ }^{[12]}$. Similarity value, $\mathrm{S}$, is calculated for each anode design using:

$$
S=\sum\left|\varphi_{c g, i}-\varphi_{n c g, i}\right|
$$

where $\varphi_{\mathrm{cg}, \mathrm{i}}$ is the weighting potential of the collecting grid at location $\mathrm{i}$ across the width of the detector and $\varphi_{\text {ncg, i }}$ is the weighting potential of the non-collecting grid at location i across the width of the detector.

The uniformity and similarity values of each anode design were then normalized and added together to calculate the FOM for each design, with a lower FOM corresponding to a better design ${ }^{[12]}$. The design will also be compared to a directly-deposited CZT detector with the same coplanar anode pattern via spectroscopic measurements. 


\section{Results and Discussion}

Figure 14 show the weighting potential generated for directly deposited electrodes and Figure 15 show the weighting potential for proximity electrodes across the detector depth. One can see that in the case of directly deposited electrodes, the result of subtracting the weighting potential of collecting electrode and the weighting potential for non-collecting electrode is equal to one at the detector surface. This is not the case in proximity electrodes where the subtracted signal is equal to approximately $0.4 \mathrm{~V}$ due to the added layers in the design. This is one of the drawbacks of using proximity electrodes. To investigate the effect of the dielectrics and their thicknesses, the detector was simulated using multiple dielectric materials at one thickness and then varying the thickness of one dielectric in the following section for directly deposited electrodes and proximity electrodes respectively.

Figures 16 and 17 show the weighting potential generated across the detector width. The shape of the two weighting potentials is different due to the addition of the aluminum guard ring in the proximity sensing design.

\section{Determining the Effects of Dielectric}

The goal of this set of simulations was to determine the effect of changing the dielectric on the weighting potential and the electric field inside the crystal. Weighting potentials and electric fields were measured $5 \mu \mathrm{m}$ below the anode. Figure 18 shows the effect of different dielectrics with the same thickness, $50 \mu \mathrm{m}$. The value of the weighting potential increases as the dielectric constant increases. Plexiglas was chosen because it has a dielectric constant close to Kapton films (3.4 vs 3.5 for Kapton). Kapton films are readily available and will be used in the implementation stage of this project.

Figure 19 show that the electric field drops as the dielectric constant increases. Even though the weighting potential improves as the dielectric constant increases, the induced charge is proportional to both the weighting potential and the electric field. Figure 11 shows the product of multiplying the weighting potential with the electric field. A higher dielectric constant will generate a worse induced signal, as expected.

The induced current was proportional to both the electric field and the weighting potential as stated in section one.

\section{Determining the Effects of Dielectric Thickness}

The goal of this set of simulations was to determine the effect of changing the dielectric thickness on the weighting potential and the electric field inside the crystal. The model used for simulation 
here included the $35 \mu \mathrm{m}$ cathode layer made of gold, CZT crystal, $50 \mu \mathrm{m}$ thick dielectric, $35 \mu \mathrm{m}$ thick coplanar copper electrodes, and Rogers 4350 PCB. Weighting potentials and electric fields were measured $5 \mu \mathrm{m}$ below the anode. The dielectric chosen here is air. The thicknesses were 1 $\mu \mathrm{m}, 10 \mu \mathrm{m}, 50 \mu \mathrm{m}, 100 \mu \mathrm{m}$, and $1 \mathrm{~mm}$. The first simulation was made without a dielectric in order to obtain a baseline of the weighting potential. Figures 21 and 22 show the effect of using different thicknesses of air on the weighting potential and the electric field respectively.

The value of weighting potential across the detector depth drops down as the thickness of the dielectric increases. This will affect the induced signal generated on proximity electrodes. The thickness of the dielectric should be minimized for better results.

The electric field across the detector also drops as the thickness of the weighting potential increases. Again, the thickness of the dielectric should be carefully chosen so that it will not affect the induced signal. There is a sudden increase to the electric field value when a $1 \mu \mathrm{m}$ dielectric is introduced to the system. The reason for this is still under investigation.

Figures 18-22, show the weighting potential and electric field for the same design of proximity electrodes but with different configurations using several dielectric materials. The optimum design would have the weighting potential equal to 1 so that the charge generated from radiation interaction inside the detector volume is fully collected. Additionally, the electric field value should be maximized to allow the electrons to travel towards the anode side as fast as possible with a low probability of being trapped. Figure 18, examines the weighting potential for a 50um thick dielectric, which is a reasonable thickness for a practical experiment once the design is finalized. Diamond has the maximum value for weighting potential but it's not realistic to build a radiation detector with a diamond due to its high cost. The electric field for the same thickness was simulated and the air had the highest electric field value. Similarly designing a detector with an air gap would be very expensive and unrealistic. Figure 20, is the result of multiplying the weighting potential and the electric field in Figures 18 and 19 to check which dielectric would result in the highest result for both variables. Figures 20 and 21, examine the use of an air gap as a dielectric with multiple thicknesses to understand the effect of changing the dielectric thickness on the weighting potential. The thinner the dielectric, the better the weighting potential value was, which also applies to the electric field value. Thus, it can be determined that the combination of the weighting potential and electric field is affected by the dielectric type for the same thickness.

\section{Conclusion}

Proximity charge sensing is a technique that can offer several distinct advantages over directly deposited electrodes when used with compound semiconductor detectors. The advantages are multiple starting with eliminating the need to directly deposit electrodes on the crystal, then, 
reducing the cost of the overall system, reducing leakage current associated with directly depositing the electrodes, and improving the position sensitivity in position sensing devices. The application of proximity electrodes to CZT detectors would afford the detection system the advantage of excellent energy resolution, room temperature operation in addition to the advantages offered by proximity electrodes.

ANSYS Maxwell simulations show that for proximity charge sensing to produce the best-induced current, an insulator with a low dielectric and a low thickness should be used so that the electric field and the weighting potential are not affected. The results revealed that the best dielectric material, which does not reduce the value of the weighting potential when added to the model, is diamond with only $20 \%$ reduction. However, it is not practical to use diamond, as it will increase the price of the detector significantly. At the same time, diamond is the worst dielectric material that affects the electric field. A 50um thick diamond will reduce the electric field to $\sim 2000 \mathrm{~V} / \mathrm{m}$ compared to $\sim 8800 \mathrm{~V} / \mathrm{m}$ for air and vacuum. Multiplying the weighting potential and the electric field results in Teflon and Plexiglas being the best dielectric materials to be implanted on the first prototype when built. In terms of the dielectric thickness, air was used to measure the response of thickness on weighting potential and the electric field. The thicknesses used were 1, 10, 50, 100, and $1000 \mu \mathrm{m}$. The weighting potential was reduced $50 \%$ in the $50 \mathrm{um}$ case but dropped three orders when the thickness increased to 100um and 1000um respectively. Even though vacuum and air have the lowest dielectric values, 1 and 1.00059 respectively, they are not recommended to be used in the first prototype because it will be very difficult to build the prototype using these dielectrics. Kapton film is recommended for the first prototype since Kapton is readily available in the market.

The simulation showed that the shape of weighting potential for the collecting and non-collecting electrodes across the detector depth does not change across the detector depth between proximity electrodes and directly deposited electrodes. However, the shape of the weighting potential for the collecting and non-collecting electrodes changes across the detector width because of the addition of the aluminum ring in the proximity electrode case.

\section{Acknowledgment}

This project was funded by the Deanship of Scientific Research (DSR) at King Abdulaziz University, Jeddah, under grant no. RG-5-135-40. The authors, therefore, gratefully acknowledge DSR technical and financial support. 


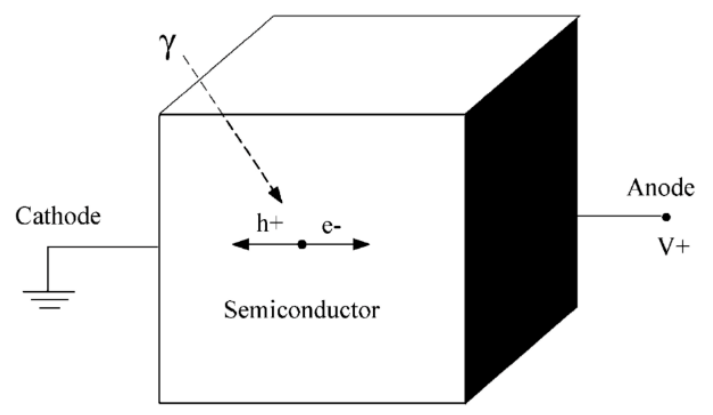

Figure 1: Basic semiconductor detector with planar electrodes ${ }^{[1]}$

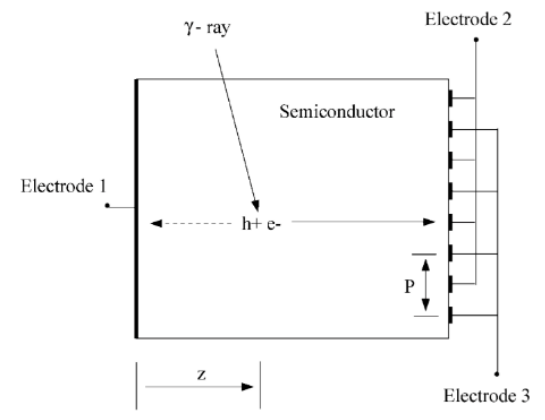

Figure 2: Coplanar grid anode pattern ${ }^{\text {[2] }}$

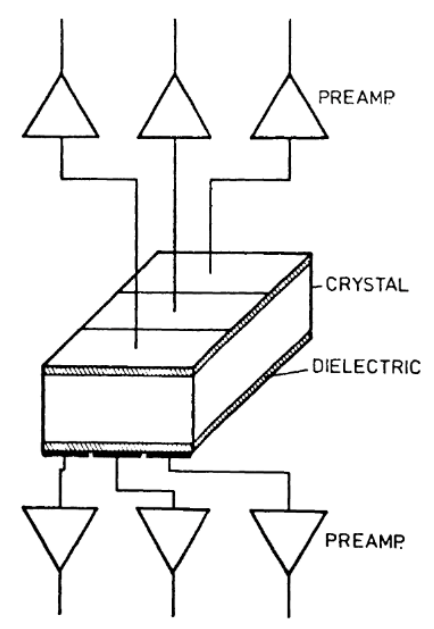

Figure 3: Two dimensional detector forming a "checker-board" configuration ${ }^{[10]}$ 


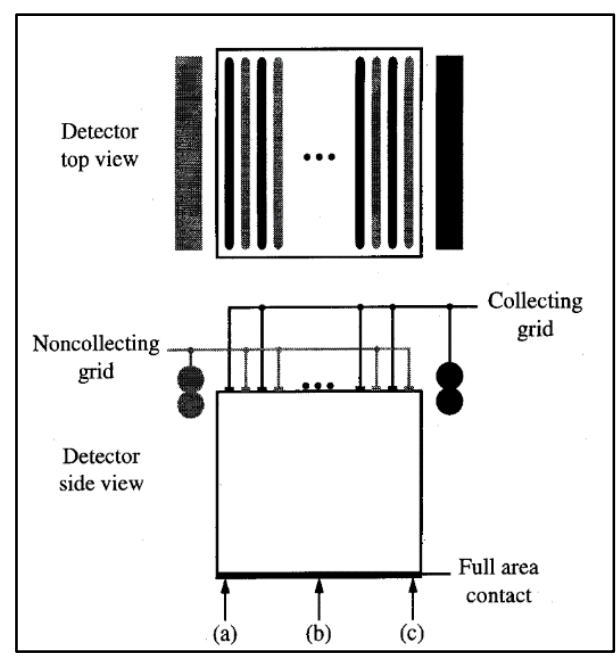

a

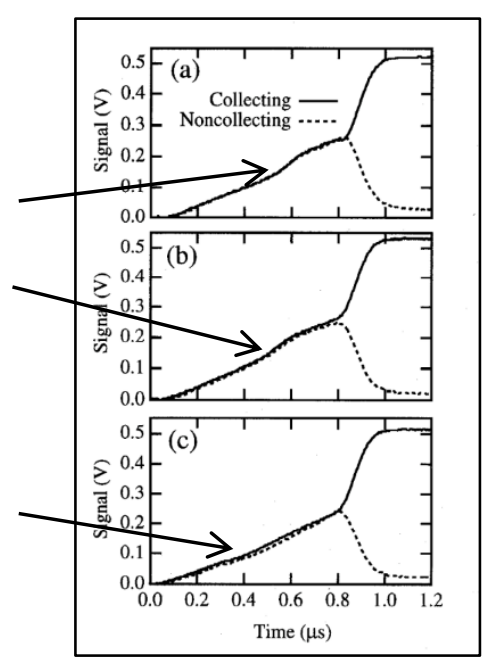

B

Figure 4: a) Schmatic diagram of a simple coplanar-grid detector with addition of externl electrodes for edge effect, b) Generated weighting potentinal for CG and NCG along the width of the detector for the three positions indicated at the bottom of 8 a) ${ }^{[11]}$

(a)

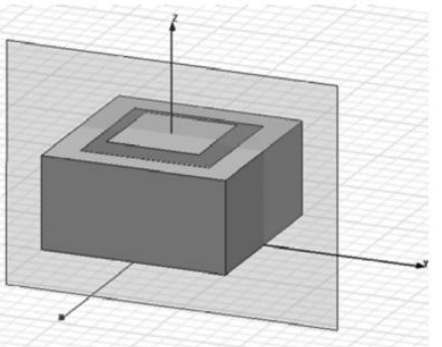

(b)

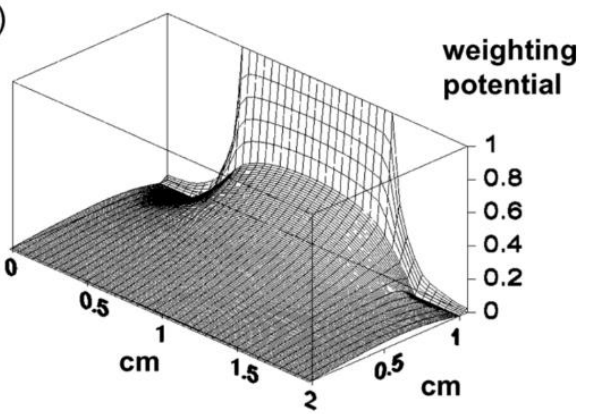

Figure 5: Geometry of the detector simulation, with center proximity electrode at $0.5 \mathrm{~mm}$ above the detector surface. (b) Calculated weighting potential of the proximity electrode along a vertical plane through the center of the detector, as shown in (a) ${ }^{[3]}$ 


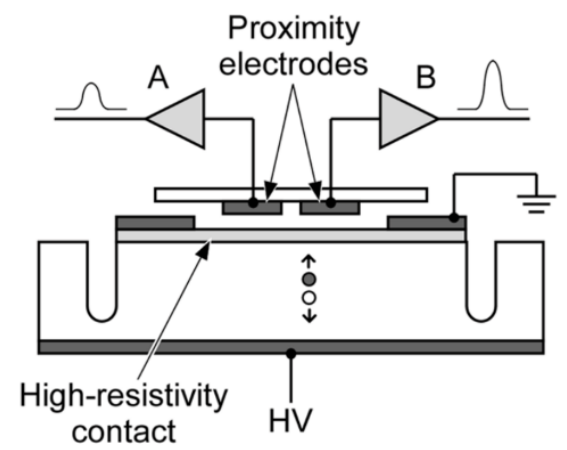

Figure 6: Non-contact readout of a detector using proximity electrodes ${ }^{[3]}$

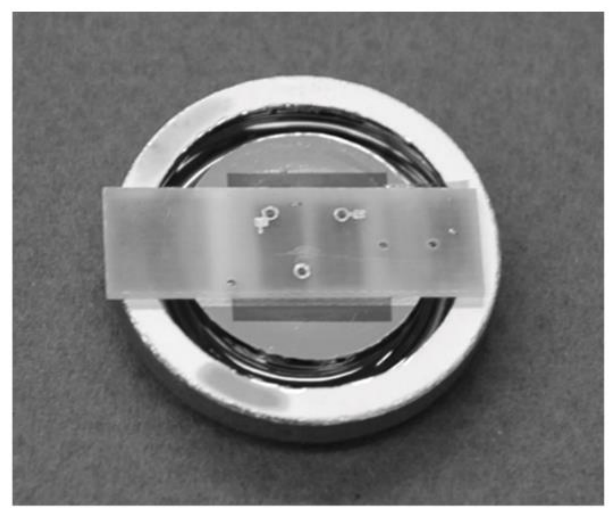

Figure 7: $\mathrm{Si}(\mathrm{Li})$ detector used to demonstrate non-contact readout with proxiity electrodes ${ }^{[3]}$

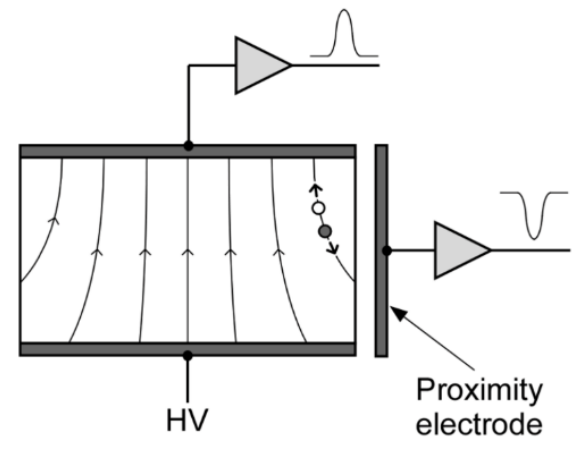

Figure 8: Sensing incomplete charge collection on the detector surface using proximity electrodes ${ }^{[3]}$ 


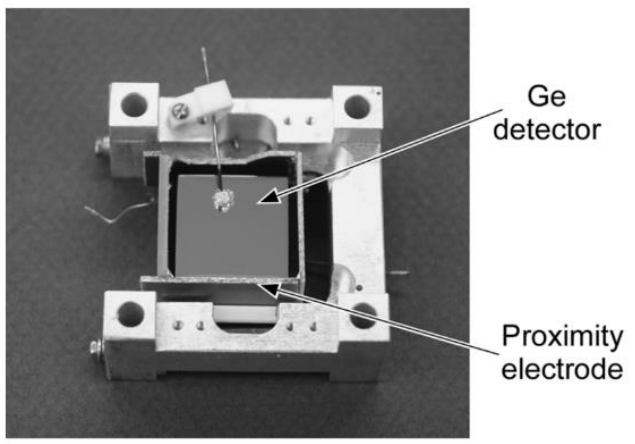

Figure 9: Ge detector with a wrap-around proximity electrode ${ }^{[3]}$

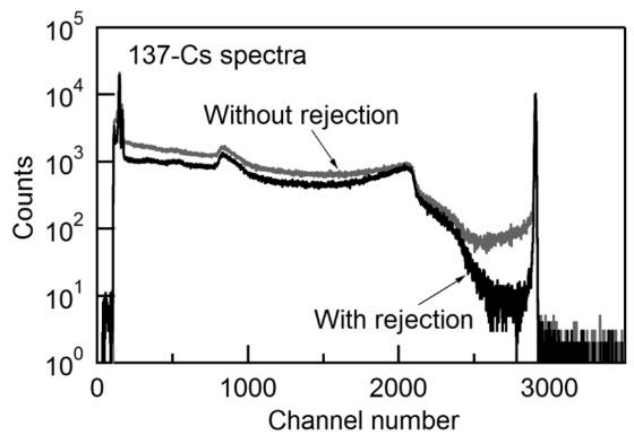

Figure 10: Reduction in spectral background achieved by rejecting surface collection events in the Ge detector using signals from the proximity electrode ${ }^{[3]}$

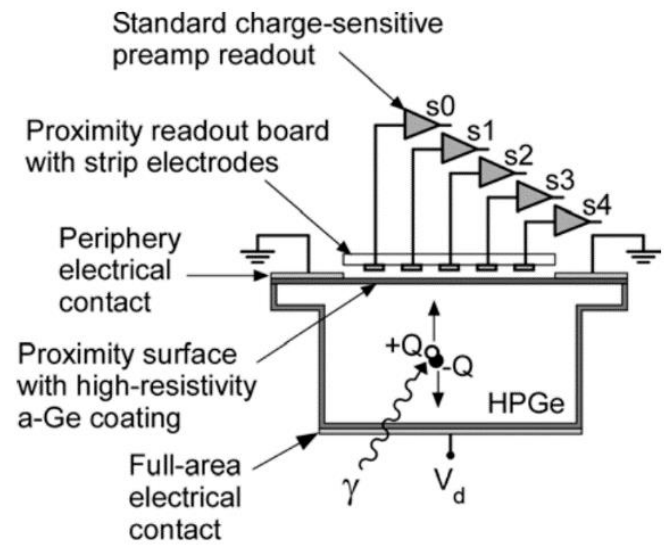

Figure 11: Schematic cross-sectional diagram of the HPGe-based proximity electrode readout detector. The readout electrodes consist of five strips oriented perpendicular to the cross-sectional plane. Not shown is a thin Kapton sheet used to set the distance between the strips and the proximity surface ${ }^{[4]}$ 


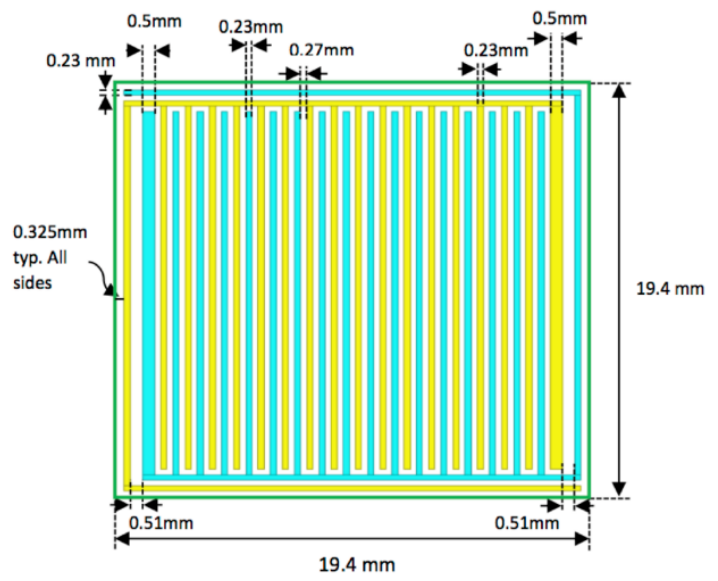

Figure 12: Coplanar pattern dimensions used in ANSYS Maxwell simulations ${ }^{[12]}$

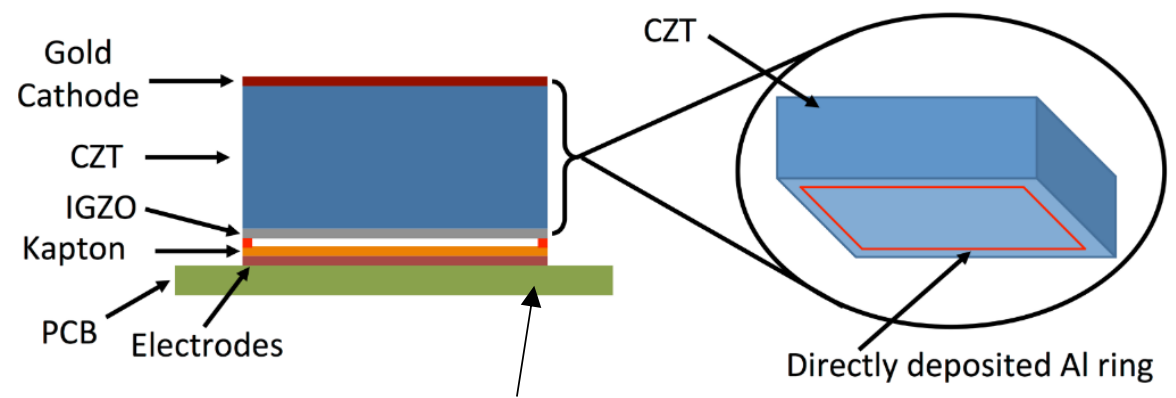

Figure 13: Proximity electrode model used for simulation in ANSYS Maxwell

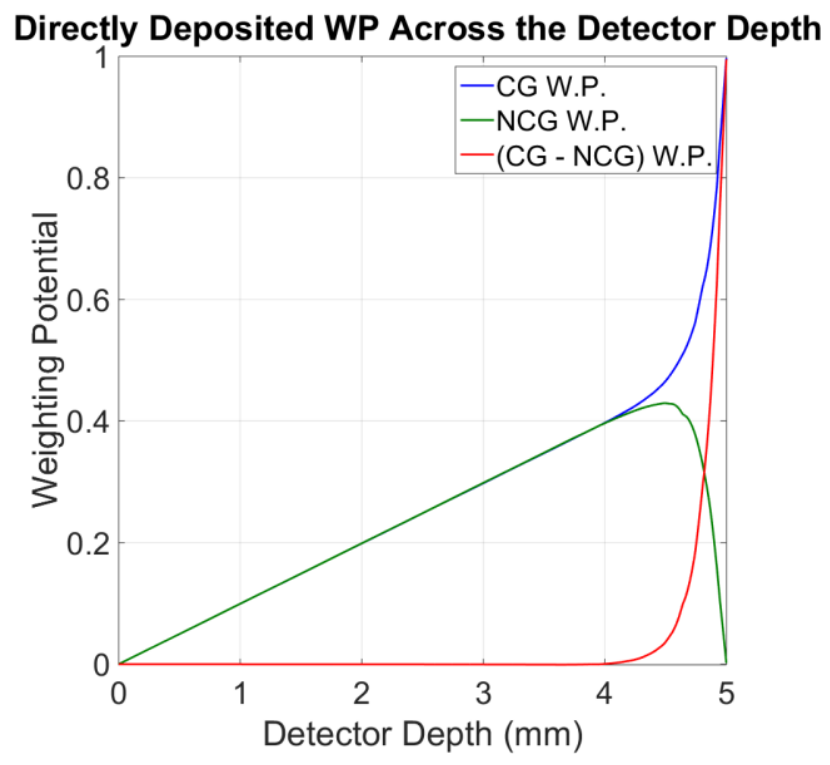

Figure 14: Generated weighting potential for directly deposited electrodes across the detector depth 


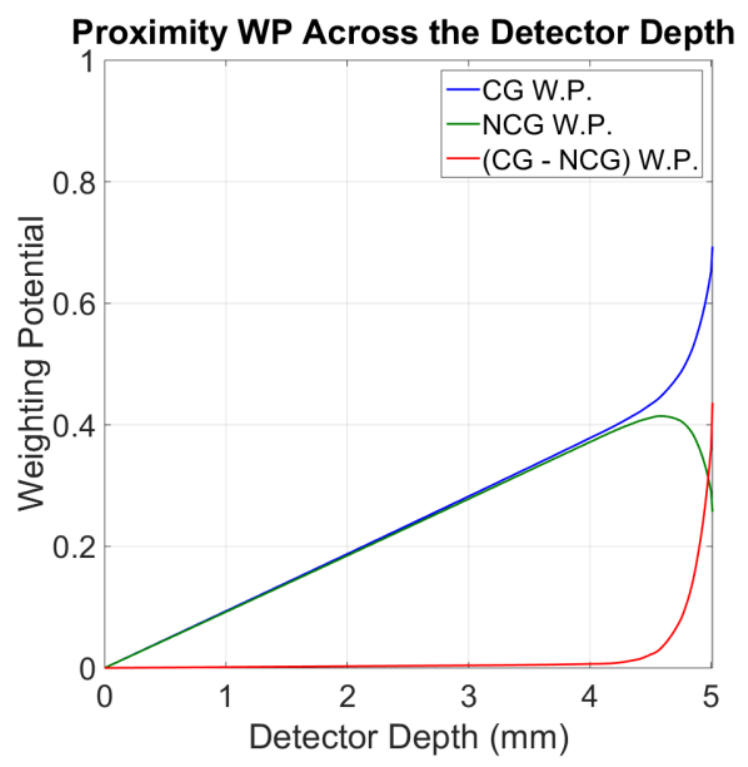

Figure 15: Generated weighting potential for proximity electrodes across the detector depth

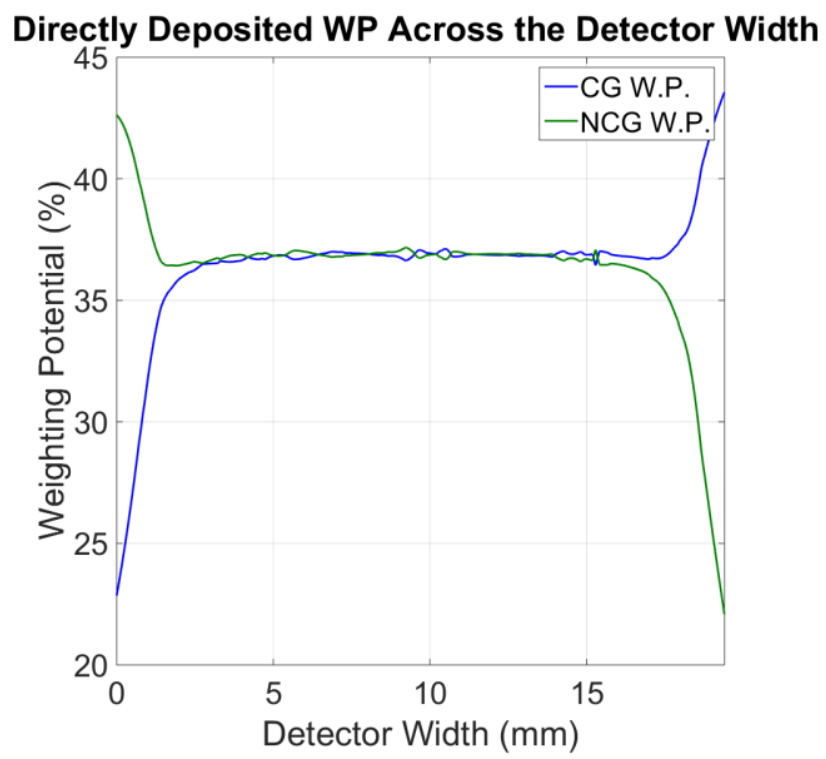

Figure 16: Generated weighting potential for directly deposited electrodes across the detector width 


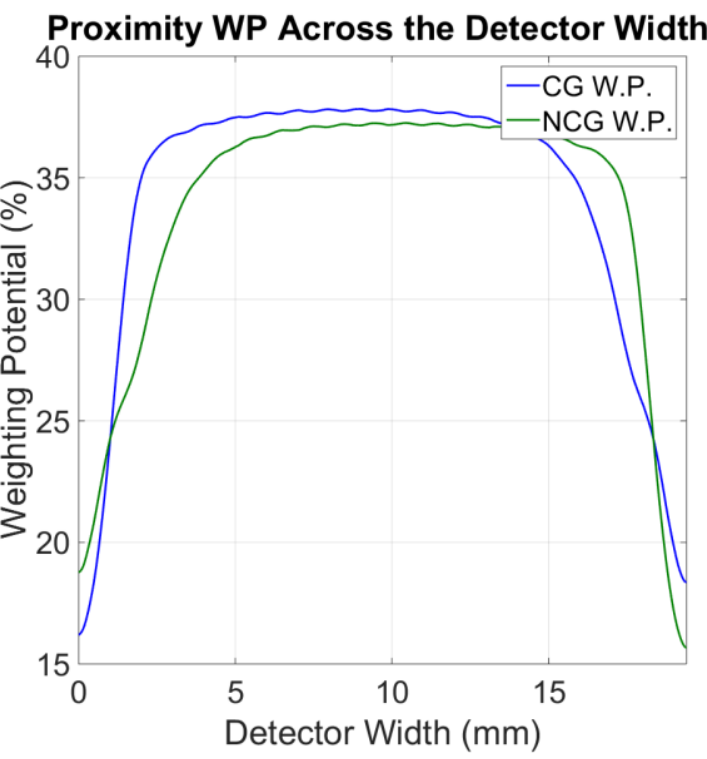

Figure 17: Generated weighting potential for proximity electrodes across the detector width

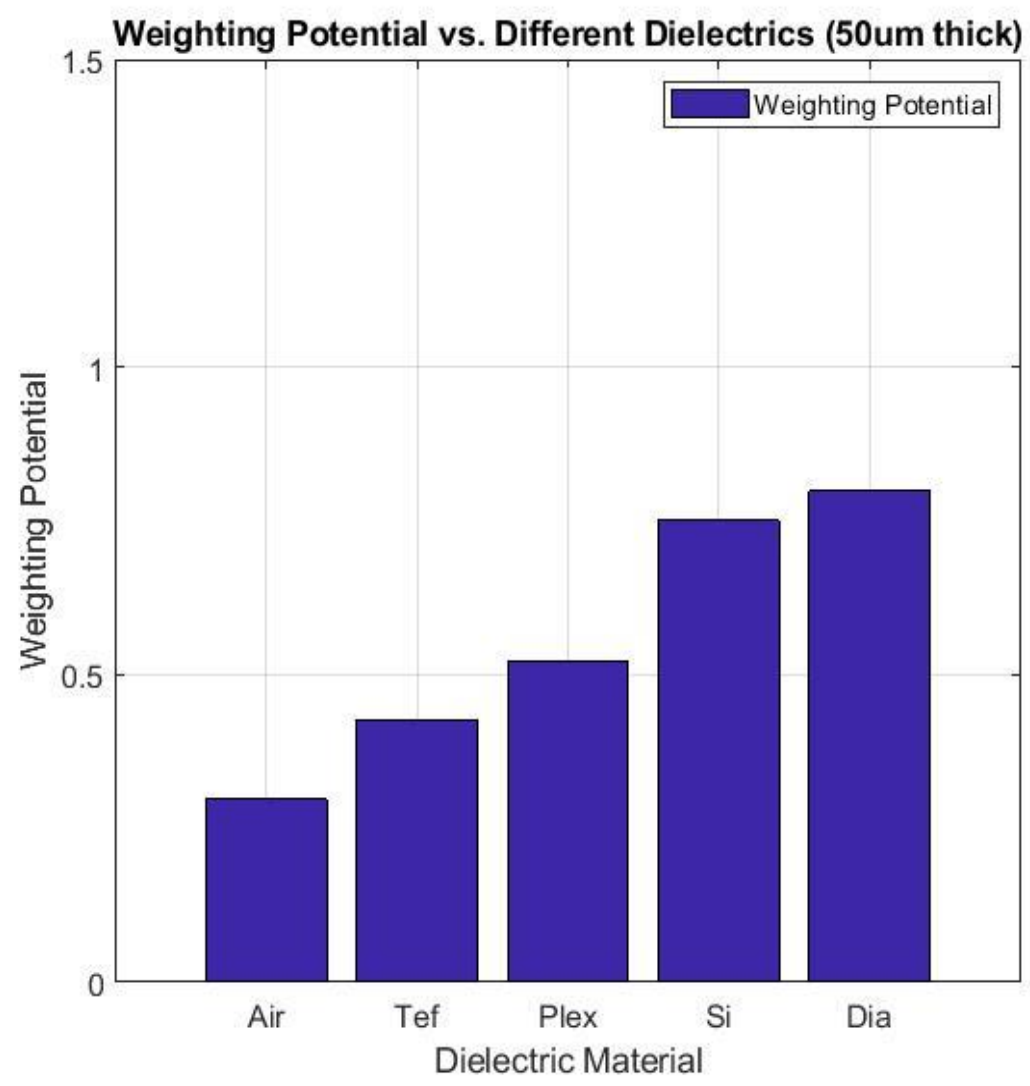

Figure 18: Generated weighting potential $5 \mu \mathrm{m}$ below the anode for different dielectrics $(50 \mu \mathrm{m}$ thick) 


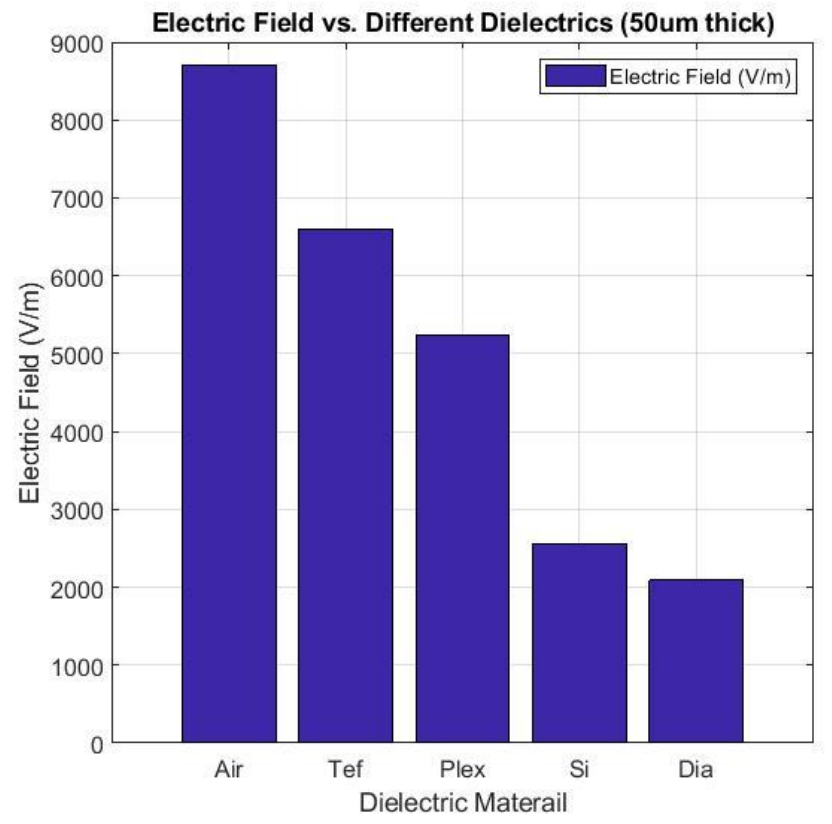

Figure 19: Generated electric field $5 \mu \mathrm{m}$ below the anode for different dielectrics $(50 \mu \mathrm{m}$ thick)

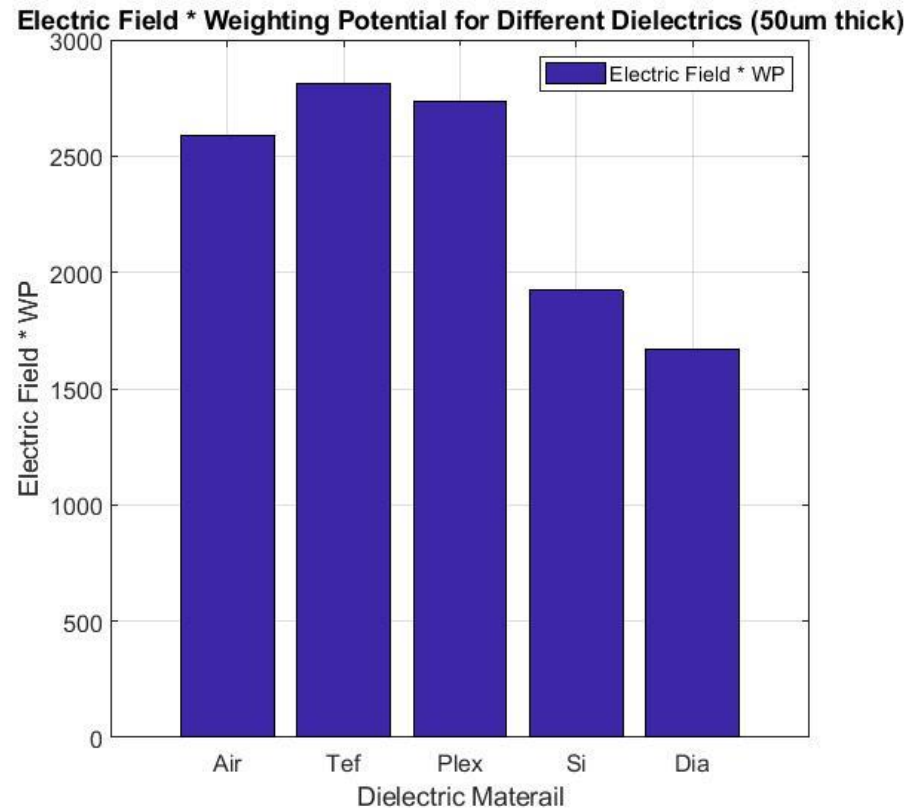

Figure 20: Weighting potential multiplied by electric field $5 \mu \mathrm{m}$ below the anode for different dielectrics $(50 \mu \mathrm{m}$ thick) 


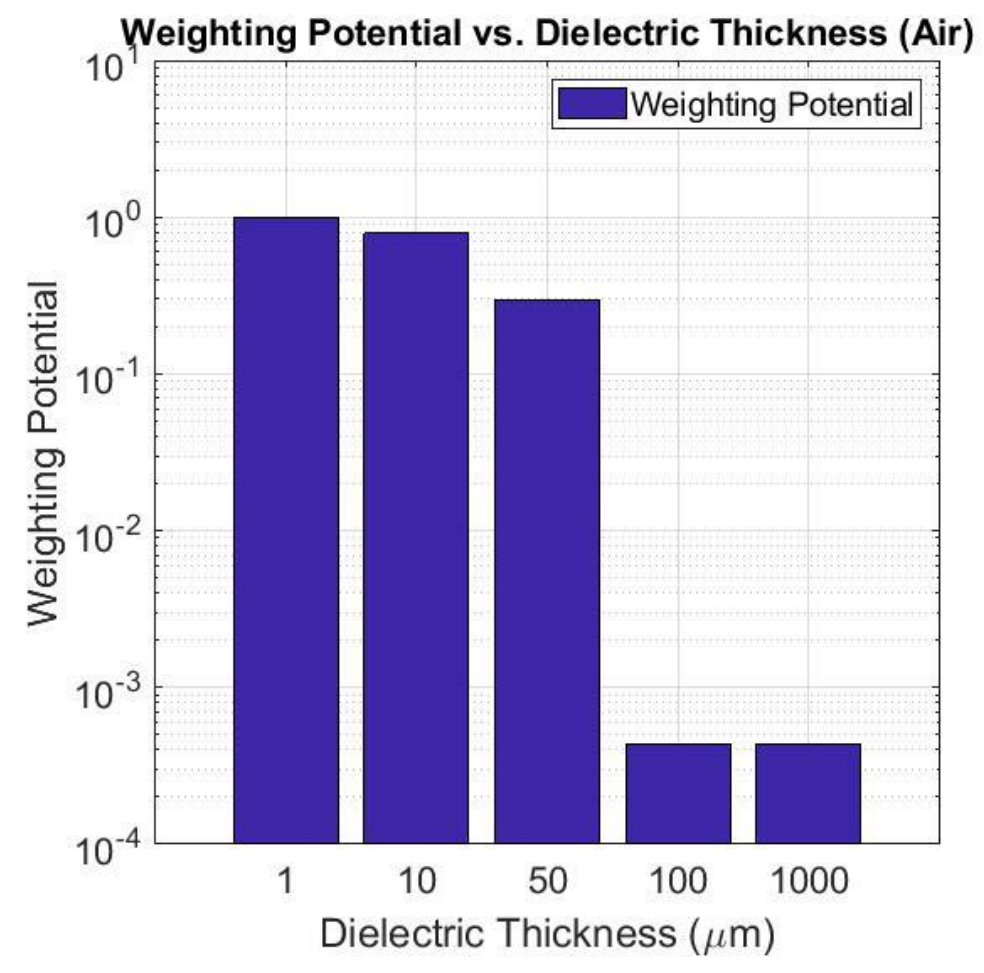

Figure 21: Generated weighting potential $5 \mu \mathrm{m}$ below the anode at different air thickness

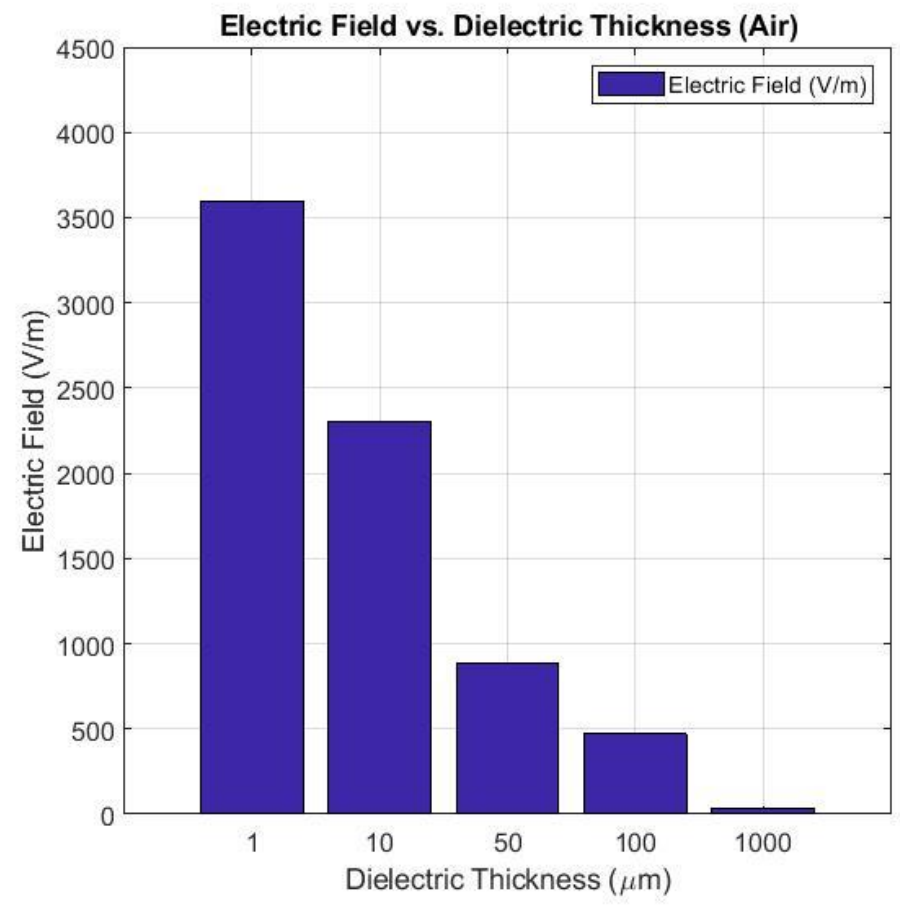

Figure 22: Generated electric field $5 \mu \mathrm{m}$ below the anode at different air thickness 
Table 1: Detector Layers in Simulation Model

\begin{tabular}{|c|c|c|c|c|}
\hline $\begin{array}{l}\text { Layers from } \\
\text { bottom to top }\end{array}$ & Material & Area $\left(\mathrm{mm}^{2}\right)$ & Thickness (mm) & Notes \\
\hline 1 & Gold & $19.4 \times 19.4$ & 0.035 & \\
\hline 2 & CZT & $19.4 \times 19.4$ & 5 & CdZnTe \\
\hline 3 & a-IGZO & $19.4 \times 19.4$ & 0.0001 & $\begin{array}{l}\text { Amorphous Indium } \\
\text { Gallium Zinc Oxide }\end{array}$ \\
\hline 4 & Kapton & $19.4 \times 19.4$ & 0.05 & \\
\hline 5 & Aluminum & $19.4 \times 1$ & 0.0002 & $\begin{array}{l}\text { Ring around crystal } \\
\text { surface }\end{array}$ \\
\hline 6 & Copper & $19.4 \times 1$ & 0.035 & $\begin{array}{l}\text { Interdigitated electrode } \\
\text { (Fig 3) }\end{array}$ \\
\hline 7 & Teflon PCB* & $19.4 \times 19.4$ & 0.503 & $\begin{array}{c}\text { Teflon Printed Circuit } \\
\text { Board }\end{array}$ \\
\hline
\end{tabular}

* Rogers 4350 PCB, a board made of Teflon that will help reduce the distortion in the electric field inside the crystal. 


\section{References}

[1] G. F. Knoll, Radiation detection and measurement. New York, NY, USA, Wiley, 2000.

[2] He, Z. (2001). Review of the Shockley-Ramo theorem and its application in semiconductor gamma-ray detectors. Nuclear Instruments and Methods in Physics Research Section A: Accelerators, Spectrometers, Detectors and Associated Equipment, 463(1-2), 250-267. Doi:10.1016/s0168-9002(01)00223-6.

[3] Luke, Paul N., et al. "Proximity Charge Sensing With Semiconductor Detectors." IEEE Transactions on Nuclear Science, vol. 56, no. 3, 2009, pp. 808-812. DOI: 10.1109/TNS.2008.2011483.

[4] Amman, Mark, et al. "Proximity Electrode Signal Readout of High-Purity Ge Detectors." IEEE Transactions on Nuclear Science, vol. 60, no. 2, 2013, pp. 1213-1218. DOI: 10.1109/TNS.2013.2247773.

[5] Hussain, A., Begum, A. and Rahman, A. (2019). Characterization of Nanocrystalline Lead Sulphide Thin Films Prepared by Chemical Bath Deposition Technique. [online] SpringerLink. Available at: https://link.springer.com/article/10.1007/s13369-012-0390-3.

[6] Altıkka, B. (2019). Effects of Inhibitor on PbS Thin Films Obtained by Chemical Bath Deposition. [online] SpringerLink. Available at: https://link.springer.com/article/10.1007/s13369015-1680-3.

[7] Wattoo, M., Tirmizi, S., Quddos, A., Khan, M., Wattoo, F., Wadood, A. and Ghangro, A. (2019). Aerosol-Assisted Chemical Vapor Deposition of Thin Films of Cadmium Sulfide and Zinc Sulfide Prepared from Bis(dibutyldithiocarbamato)Metal Complexes. [online] SpringerLink. Available at: https://link.springer.com/article/10.1007/s13369-011-0060-x.

[8] Badran, R. (2019). The Effect of Helium Dilution on Optical and Photoelectric Properties of a-Si: H Thin Films Prepared by Plasma Enhanced Chemical Vapor Deposition Technique. [online] SpringerLink. Available at: https://link.springer.com/article/10.1007/s13369-011-0147-4.

[9] El Khalfi, A., Ech-chamikh, E., Ijdiyaou, Y., Azizan, M. and Essafti, A. (2019). Optical Properties of Amorphous Silicon-Carbon Alloys (a-Si x C1-x) Deposited by RF Co-Sputtering. [online] SpringerLink. Available at: https://link.springer.com/article/10.1007/s13369-014-1134-3.

[10] Kurz, R. E., et al. "Position-Sensitive Semiconductor Detector for Gamma Rays." IEEE Transactions on Nuclear Science, vol. 24, no. 1, 1977, pp. 255-259.

[11] Luke, P.N., et al. "Electrode design for coplanar-grid detectors." IEEE Transactions on Nuclear Science, vol. 44, no. 3, 1997, pp. 713-720.DOI: 10.1109/23.603739. 
[12] Ranjbar, L., Farsoni, A. T., \& Becker, E. M., Lee Chee Sing, and Czyz, S. A, "Evaluation of a two-channel coplanar CZT detector prototype for atmospheric radioxenon measurement" IEEE Nucl. Sci. Symp. \& Med. Img. Conference, Nov-Oct, 2015. 